\title{
Implementation of Plug-in Type Repetitive Controller for Position-based Periodic Control Systems
}

\author{
Ge-Liang Hsu, Wu-Sung Yao, and Mi-Ching Tsai* IEEE Senior Member
}

\author{
Department of Mechanical Engineering, \\ National Cheng Kung University, \\ Tainan 701, Taiwan, ROC.
}

\begin{abstract}
Position-based periodic motions have appeared in many industrial processes such as cam-followers. The period of the output motion driven by the cam varies in the time-domain but is fixed in the angular position-domain, which is called time-varying periodic motion. This paper presents a plug-in type repetitive control scheme for reducing tracking errors via position-based periodic reference signals and/or disturbances. Two kinds of control strategies, namely disturbance feed-forward and disturbance rejection control, are proposed to investigate control performance with time-varying periodic disturbances. The implementation technique utilized in this position-based repetitive controller is discussed in detail, and an anti-vibration control system with position-base load disturbances generated by a cam is realized. Experimental results are given to demonstrate that the repetitive control can effectively eliminate the steady-state errors within a few cycles caused by time-varying periodic disturbances. This study offers an alternative method for realizing a repetitive controller to track and/or reject periodic signals under variable periods.
\end{abstract}

Keywords: time-varying periodic, angular position domain, repetitive controller, antivibration control

\section{INTRODUCTION}

It is well known from the internal model principle [2] that the control output of a stable feedback system can track a class of reference commands without steady-state errors, if the loop transfer function model is involved with these command signals in the closed-loop system. Nowadays, a number of repetitive control algorithms have been developed (for example see [1] [6] [7] [8] [9] [10] [12] [13] [20] [21]) to reduce the steady-state errors; these algorithms govern time-base periodic reference and/or disturbance signals. A modified repetitive control system was developed in our previous study [21] (see Fig. 1), where the period $T_{d}$ of the input signal is given. The design parameters $K_{q}(s)$ and $K_{b}(s)$ are introduced to improve the closed-loop bandwidth. This repetitive controller adopts the plugin type scheme since the implementation can be easily accomplished. Note that a repetitive controller consisting of a periodic signal generator must have an integer number of samples in each period time so 
that its period is synchronized with the input signal period.

Besides time-based motion control, position-based periodic motions appear in many industrial processes and applications such as cam-follower systems, cammachining processes, and other corresponding tracking tasks [4] [[7] [8] [9] [11] [14] [17] [19]. For example, the cam machining in [22] is required to keep the removal rate constant during cam finishing in which the cam is driven at a specified variable velocity and the signal is periodical in angular position domain [17] [19]. Hereafter, this kind of motion profile is called the time-varying periodic signal, in that the period defined in the angular position domain is constant but is variable in the time domain. A simple example is given as $r(t)=\cos \left(2 \pi t^{2}\right)$ which is obviously not a timebased periodic function as shown in Fig. 2(a). However, the signal $\tilde{r}(\theta)=\cos (\theta)$ with $\theta=2 \pi t^{2}$ is a position-based periodic function with a constant period of $2 \pi$ as shown in Fig. 2(b).

In practice, if the period of the reference signal in a control system consists of time-varying functions, the conventional time-based repetitive controller is not directly applicable. Therefore, a number of adaptive repetitive control algorithms have been developed (for example, see [2] [10] [15] [18] [22] [23]) for time-varying periodic signals, which involve either the domain transformation or the period estimation technique. Rothbart [17] transformed the signal mode from the time domain to the position domain in order to eliminate angular position-dependent disturbances in constant-speed rotation control systems. In such a case, the positiondependent disturbances become periodic functions in position domains with fixed periods. The techniques based on the domain transformation can be found in [3] [15] [24] [25] [29]. However, linear timeinvariant (LTI) models often become linear time varying (LTV) after the transformation. Srinivasan and Shaw [4] developed analysis and synthesis methods for these systems using performance measures in the frequency domain such as the sensitivity function and complementary sensitivity function. Manayathara [16] proposed a discrete-time repetitive controller for the rejection of periodic load disturbances with an unknown period in a continuous steel casting process. A recursive scheme was applied to identify the unknown disturbance period and to adaptively modify the period of the repetitive control algorithm. The investigated subject can be seen in [4] [10] [16] [26] [27] [28]. However, the precision of the estimated period influences the performance of the repetitive control. Besides, a given stability with the adaptive algorithm is also required. The repetitive control often makes the design process complex.

This paper implements the plug-in type repetitive controller to track time-varying periodic signals with fixed sampling rates. In fact, the proposed approach can deal with both time-constant and time-varying periods based on the synchronization between time and position. An anti-vibration control system, which creates time-varying periodic disturbances, is constructed for experimental studies, where two control strategies are investigated, namely disturbance feed-forward and disturbance rejection control.

\section{IMPLEMENTATION OF THE PROPOSED REPETITIVE CONTROLLER}

Due to the implementation of the time delay unit, such as $e^{-s T_{d}}$ in Fig.1, a technique to realize the time delay unit for a repetitive controller is introduced with the varying period $T_{d}$ where the number of samples per period may be non-integers with a fixed sampling rate. Practically, the delay time of the repetitive control should be synchronized with the period of the input signal. Obviously, the input data is stored in storage memory and released after the given delay time; meanwhile, the memory of the released data would restore the new data in the next sampling. Thus, the key techniques to implement a time delay unit contain two major components: the memory register and a decision rule. Note that the memory registers are used to guide the input data to correct memory addresses, and the decision rule judges the sampling instant to output the delayed data. For example, if the data is delayed for a sampling time of 4 , the data will be arranged in a specific memory address which will be computed by the time counter. When the system time clock achieves the fourth sampling time, the decision rule will be enabled and then the stored data will be released. However, these implementations are not only achieved by using the time counter information, but also by using the position encoder information to deal with the influence of the position-base periodic signals. Thus, the position-based implementation of the memory register and a decision rule are named as position delay and position synchronization, which are addressed the following.

The position delay, which releases the past data at a certain cam position, is illustrated as

$$
\begin{cases}y(\theta)=0 & \text { if } \theta<P_{d} \\ y(\theta)=r_{s}\left(\theta-P_{d}\right) & \text { if } \theta \geq P_{d}\end{cases}
$$

where $y(\theta)$ is the output of position-base delay unit, $r_{S}(\theta)$ is the displacement of cam devices, $\theta$ is the rotational position, and $P_{d}$ is the radian of the position delay, which is usually $2 \pi$.

Let $k \Delta t \quad(k=1,2,3 \ldots)$ denote the discrete-time instant where $k$ is the counter and $\Delta t$ is the sampling time. The position synchronization is implemented as

$$
m(i)=r_{s}(\theta) \text { if } \theta(k \Delta t) \geq k_{\theta} \Delta \theta
$$

where $m(i)$ is the memory range; $i(=1,2,3 \ldots n)$ represents a memory address, $n$ is the size of the 
buffer memory; $k_{\theta}(=1,2,3 \ldots)$ is the sampling position counter; and $\Delta \theta$ is the rotational position sampling.

\section{AN ILLUSTRATED EXAMPLE}

An anti-vibration control system is examined in this paper for experimental study, and two control strategies of both disturbance feed-forward control and disturbance rejection control are investigated to verify the proposed repetitive control scheme.

\subsection{Experimental Setup}

The experimental setup of an anti-vibration control system is displayed in Fig.3. A linear servomotor (Fig.3(a)) is given to provide the control force. Figure 3(b) shows a passive suspension unit of the main structure. A position-based periodic disturbance generator is shown in Fig.3(c), which consists of a reduction device, a follower, and an eccentric wheel driven by a servo motor. In the case, the control target is to reduce the vibration of the load in Fig.3(d) caused by a periodic disturbance.

Assume that the elasticity and damping effects of the cam-follower system in Fig.3(c) are ignored. The control system in Fig.3 can be simplified to a second order dynamic system as depicted in Fig.4, where $F_{\text {com }}$ denotes the compensation force caused by the linear motor, $M_{l}$ is the equivalent mass of the load, $M_{b}$ is the mass of the cam-follower structure, $K$ is the elasticity coefficient, $B$ is the damping coefficient, $r_{l}$ and $r_{b}$ are the displacements of the load and the follower, respectively. Then the dynamic equations of the system are given by

$$
\left\{\begin{array}{l}
M_{l} \ddot{r}_{l}=-K\left(r_{l}-r_{b}\right)-\xi\left(\dot{r}_{l}-\dot{r}_{b}\right)+F_{a} \\
M_{b} \ddot{r}_{b}=K\left(r_{l}-r_{b}\right)+\xi\left(\dot{r}_{l}-\dot{r}_{b}\right)-F_{a}
\end{array}\right.
$$

The block diagram of the control system is depicted in Fig.5, where $F_{D}$ denotes the disturbance force caused by the displacement of the follower in Fig.3(c). The parameters of the experimental setup are $M_{l}=21.76 \mathrm{Kg}, \quad K=6726.3 \mathrm{Nt} / \mathrm{m}$, $\xi=768.41 \mathrm{Nt} / \mathrm{m} / \mathrm{s}$, and $v$ is the velocity between the $M_{l}$ and $M_{b}$ in the illustrated example.

\subsection{Control Strategy for Vibration Cancellation}

In this paper, the disturbance feed-forward control (Fig. 6) and the disturbance rejection control (Fig. 7) are proposed in the illustrated example of the active anti-vibration control. The position loop and velocity loop controllers, P+PDFF (proportional + pseudo derivative feedback feed-forward) [5], are predesigned to stabilize the control system for the required performance. According to the design rule [21] and the system model (Fig.5), the parameters of the plug-in type repetitive controller can be obtained, which are given in Table 1.
In Fig. 6, the purpose of the disturbance feed-forward control is to create the inverse motions which are used to reduce the original motions of the load. $G_{p}(s)$ is the control plant (see Fig.5) and $E(s)$ represents LAE (Low Acceleration Estimator) to estimate the relative velocity between the load and the linear motor. The disturbance force $F_{D}$ is a function of the displacement $r_{b}(\theta)$ and the follower, such as

$F_{D}=K r_{b}(\theta)+B \frac{d r_{b}(\theta)}{d \theta}$

and the desired position delay $P_{d}$ is illustrated as

$P_{d}=360-\omega_{r}\left(T_{q}+T_{b}\right)$

where $\omega_{r}$ is the velocity command.

In the disturbance feed-forward control, the motion command can be generated easily to follow the displacement $\left(r_{b}(\theta)\right)$ in simulation. However, in experimentation, an initial position of the disturbance generator must be the same as the initial position of $r_{b}(\theta)$, which is difficult to be realized.

The disturbance rejection control, shown in Fig.7, is a position control with a zero command input. Compared to the disturbance feed-forward control, the disturbance rejection control does not require the initial position of the disturbance generator, which means a more convenient implementation than that of the disturbance feed-forward control.

\subsection{Experiment Results}

The experimental setup is revealed as Fig.3 where two linear encoders provide the position information of the load and the linear motor, respectively, and a rotary encoder is used to measure the angular position of the eccentric wheel. At first, the disturbance feed-forward control and the disturbance rejection control, respectively, are addressed by a time-constant periodic signal input. Figure 8 shows the experimental results of two control schemes without the repetitive controller, and the results with the repetitive controller are shown in Fig.9. As can be seen, the disturbance rejection control shows a better performance than that of the disturbance feedforward control due to the nonlinear properties of the physical equipment, i.e. the passive suspension device. In the following, the disturbance rejection control is studied in real-time due to a time-varying periodic signal while the position-base delay and positional synchronization are implemented.

For the periodic signals generated by the disturbance generator, Fig.10 exhibits the response via the position-base delay method only where the vibration is still found due to non-synchronization between the memory register and the angular position, which means the memory buffer size varies. Since the velocity of the eccentric wheel is constant, the vibration is converged under the uniform width of the memory buffer. With the position-base delay and the 
positional synchronization, the vibration response is improved significantly as shown in Fig.11. The results have shown that the presented repetitive controllers can effectively eliminate the steady-state errors within only a few cycles. Note that the position delay and the position synchronization are indeed indispensable for the repetitive control while the system is involved with time-varying periodic signals.

\section{4, CONCLUSION}

This paper has investigated repetitive control implementation for tracking and/or rejecting timevarying periodic signals and presented some related implementation techniques for the delay unit, the position-base delay, and positional synchronization. Two control strategies, namely the disturbance feedforward and the disturbance rejection have been proposed in the experimental studies based on an anti-vibration control system. The measured results demonstrated that the proposed repetitive controller can effectively eliminate the steady-state errors due to time-varying periodic disturbances within a few cycles.

\section{REFERENCES}

[1] H. L. Broberg and R. G. Molyet (1994). A new approach to phase cancellation in repetitive control. Proceedings of the 29th IEEE IAS, pp. 1766-1770.

[2] Z. Cao and G. Ledwich (2001). Tracking variable periodic signals with fixed sampling rate. Proceedings of the 40th IEEE Conference on Decision \& Control, Orlando, Florida USA, December.

[3] C. L. Chen and G. T. C. Chiu (2004). Compensating for spatially repetitive disturbance with linear parameter varying repetitive control. Proceedings of the IEEE International Conference on Control Applications, Taipei, Taiwan, vol.1, pp.736-741.

[4] H. Dotsch and H. Smakman (1995). Adaptive repetitive control of a compact disc mechanism. Proceedings of the 34th Conference on Decision \& Control, New Orleans, LA USA, December.

[5] G. Ellis and R. D. Lorenz (1999). Comparison of Motion Control Loops for Industrial Applications. Proceedings of the IEEE Conference on Industry Applications, vol.4, pp.2599 - 2605, Phoenix, AZ, USA.

[6] S. Hara, Y. Yamammoto, T. Omata, and M. Nakano (1988). Repetitive control system: a new type servo system for periodic exogenous signals," IEEE Transactions on Automatic Control, vol. 33, no. 7 , pp. 659-666.

[7] G. Hillerstrom and J. Sternby (1993). Application of repetitive control to a peristaltic pump. Proceedings of the Automatic Control Conference, pp. 136-140.

[8] T. Inoue (1990). Practical repetitive control system design. Proceedings of the 29th
Conference on Decision and Control, vol. 3, pp. 1673-1678.

[9] M. Ishida, S. Higuchi, and T. Hori (1994). Reduction control of mechanical vibration of an induction motor with fluctuated torque load using repetitive controller. Proceedings of IEEE International Conference on Industrial Technology, pp. 533-537.

[10] T. Jolly, T. C. Tsao and J. Bentsman (1993). Rejection of unknown periodic load disturbances in continuous steel casting process using learning repetitive control approach. Proceedings of IEEE International Symposium on Intelligent Control, pp. 36-41.

[11] K. Kanzaki and N. Kobayashi (1989). Output of cam motion curve by DC servo motor application of inverse systems. Journal of the Japan Society for Precision Engineering, vol.55, no.12, pp.2215-2221.

[12] C. Kempf, W. Messner, M. Tomizuka, and R. Horowitz (1992). A comparison of four discretetime repetitive control algorithms. Proceedings of the American Control Conference, Chicago, IL, June, vol. 4, pp. 2700-2704.

[13]F. Kobayashi, S. Hara, and H. Tanaka (1990). Reduction of motor speed fluctuation using repetitive control. Proceedings of the 29th CDC, pp. 1697-1702.

[14] M. Labarre and B. Schneider (1995). Electronic cams boost machine flexibility, productivity. Power Transmission Design, vol.37, iss.3, pp.3538.

[15]Z. H. Luo and B. Mahawan (1998). Repetitive control of tracking systems with time-varying periodic references. Proceedings of the 37th IEEE Conference on Decision \& Control, Tampa, Florida, USA.

[16] T. J. Manayathara, T. C. Tsao and J. Bentsman (1996). Rejection of unknown periodic load disturbances in continuous steel casting process using learning repetitive control approach. IEEE Transactions on Control Systems Technology, vol.4, pp.259-265.

[17]F. A. Rothbart (1956). Cams-design, dynamics and accuracy, John Wiley and Sons, New York.

[18] J. H. She and M. Nakano (1996). Elimination of position-dependent disturbances in constantspeed-rotation control systems. Proceedings of the 35th Conference on Decision \& Control, Kobe, Japan.

[19]D. Tesar and G. K. Matthew (1976). The Dynamics Synthesis, Analysis, and Design of Modeled Cam Systems, Lexington Books.

[20] M. Tomizuka, T. C. Tsao, and K. K. Chew (1988). Discrete-time domain analysis and synthesis of repetitive controllers. Proceedings of the American Control Conference, vol. 2, Atlanta, Ga., pp. 860-886.

[21] M. C. Tsai and W. S. Yao (2002). Design of a plug-in type repetitive controller for periodic inputs. IEEE Transactions On Control Systems Technology, vol. 10, no. 4, pp. 547-555. 
[22] T. C. Tsao and M. Tomizuka (1988). Adaptive and repetitive digital control algorithms for noncircular machining. Proceedings of the American Control Conference, Atlanta, Ga. vol. 1, pp. 115-120.

[23] T. C. Tsao and Y. X. Qian (1993). An adaptive repetitive control scheme for tracking periodic signals with unknown period. Proceedings of the American Control Conference, San Francisco, California, vol. 2, pp. 1736-1740.

[24] J. Wang and T. C. Tsao (2004). Repetitive control of linear time varying systems with application to electronic cam motion control. Proceedings of American Control Conference, Boston, Massachusetts, USA, pp.3794-3799.

[25] J. Wang and T. C. Tsao (2005). Laser beam raster scan under variable process speed - an application of time varying model reference repetitive control system. IEEE/ASME International Conference on Advanced Intelligent Mechatronics, Monterey, California, USA, pp.1233-1238.

[26] C. Zhenwei and G. F. Ledwich (2002). Adaptive repetitive control to track variable periodic signals with fixed sampling rate. IEEE/ASME Transactions on Mechatronics, vol.7, pp.378384.

[27]C. Zhenwei and G. Ledwich (2001). Tracking variable periodic signals with fixed sampling rate. Proceedings of the IEEE Conference on Decision and Control, vol.5, pp.4885-4890, Orlando, FL, USA.

[28] C. Zhenwei and G. Ledwich (2005). Tracking Variable Periodic Signals with Fixed Sampling Rate - Feedforward Control. IEEE International Conference on Industrial Technology, pp.142-145, Hong Kong.

[29] S. Zongxuan (2004). Tracking or rejecting rotational-angle dependent signals using time varying repetitive control. Proceedings of American Control Conference, vol.1, pp.144149, Boston, Massachusetts, USA.

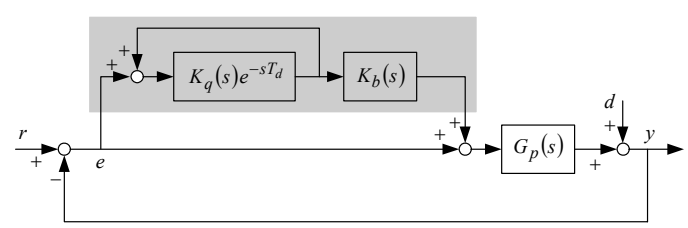

Fig. 1 A plug-in type repetitive control system
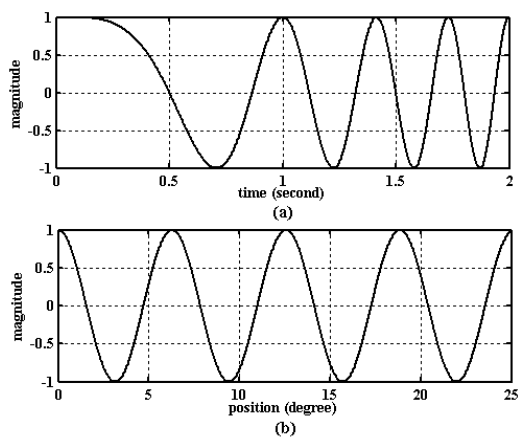

Fig. 2 An example of time-varying periodic signals. (a) the time domain plot and (b) the position domain plot

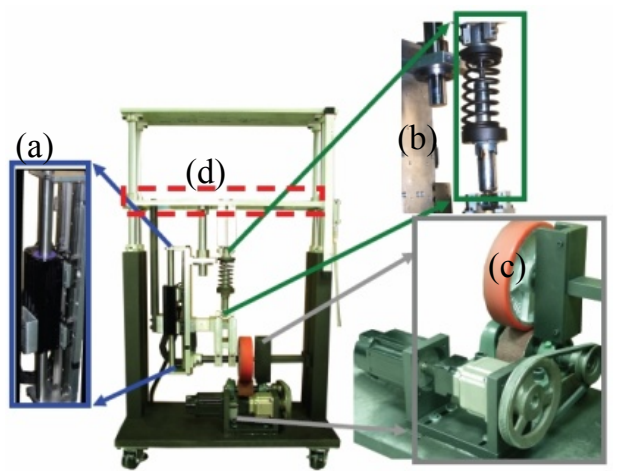

Fig. 3 The experimental setup

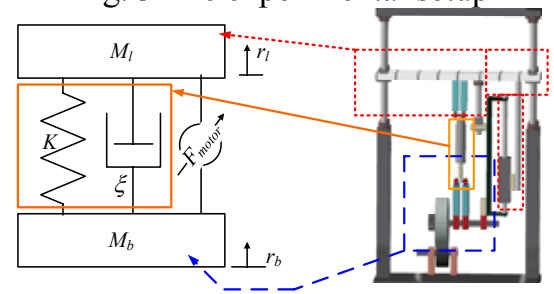

Fig. 4 The simplified model

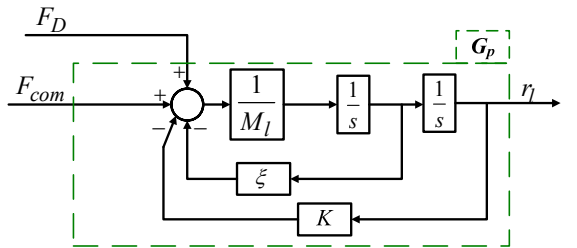

Fig. 5 The block diagram of the simplified model

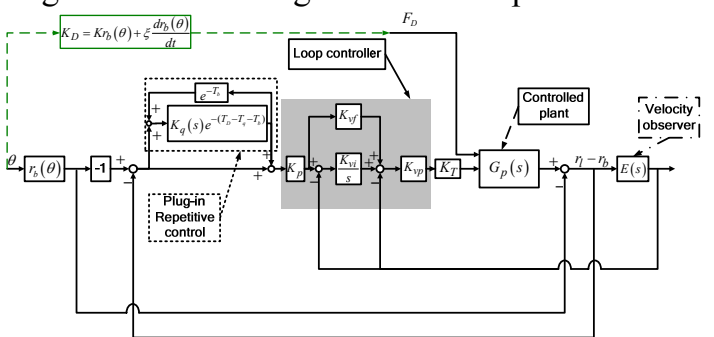

Fig. 6 The block diagram of the disturbance feedforward control 


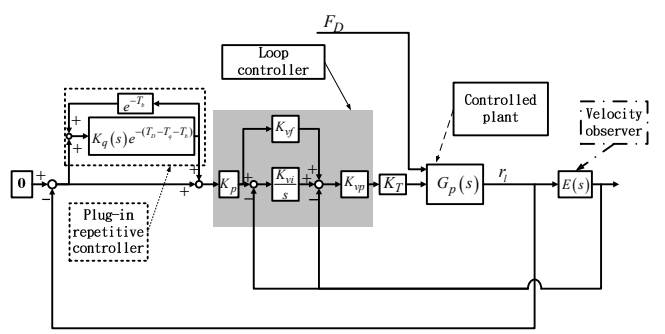

Fig. 7 The block diagram of the disturbance rejection control
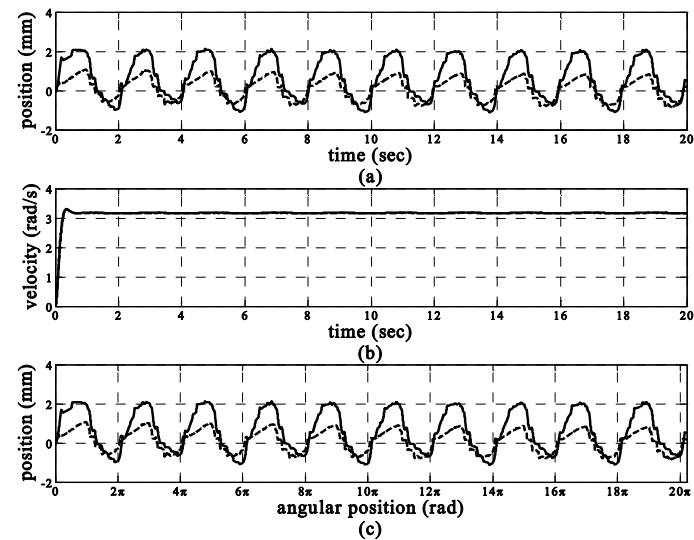

Fig. 8 Experimental results without repetitive controller $\left\{\begin{array}{l}- \text { : disturbanc feedforward } \\ -- \text { : disturbanc rejection }\end{array}\right.$. (a) The position response of the load in the time-base; (b) the velocity profile of the eccentric wheel; (c) the position response of the load in the position-base.
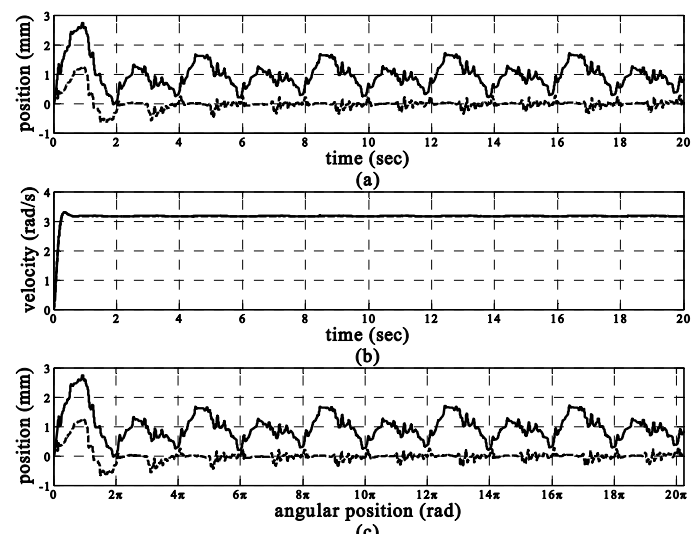

(c)

Fig. 9 The experimental results with repetitive controller $\left\{\begin{array}{l}-: \text { disturbanc feedforward } \\ --: \text { disturbanc rejection }\end{array}\right.$. (a) The position response of the load in time domain; (b) the velocity profile of the eccentric wheel; (c) the position response of the load in position domain.
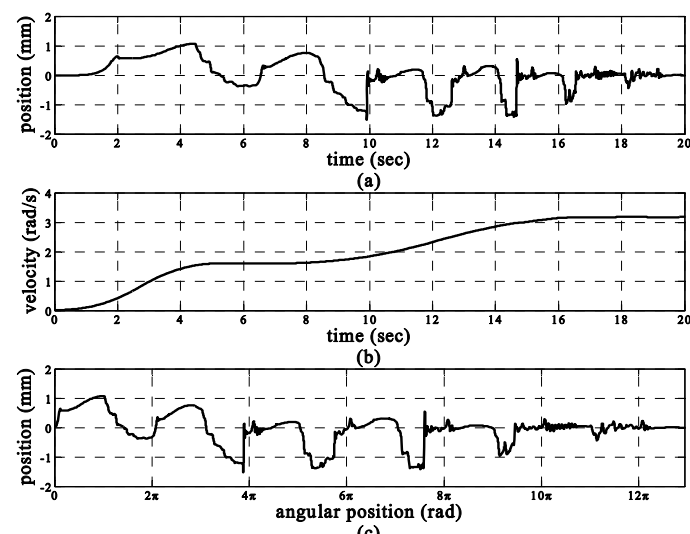

Fig. 10 The experimental results with repetitive controller using only position delay. (a)The position response of the load in time domain; (b) the velocity profile of the eccentric wheel; (c) the position response of the load in position domain.
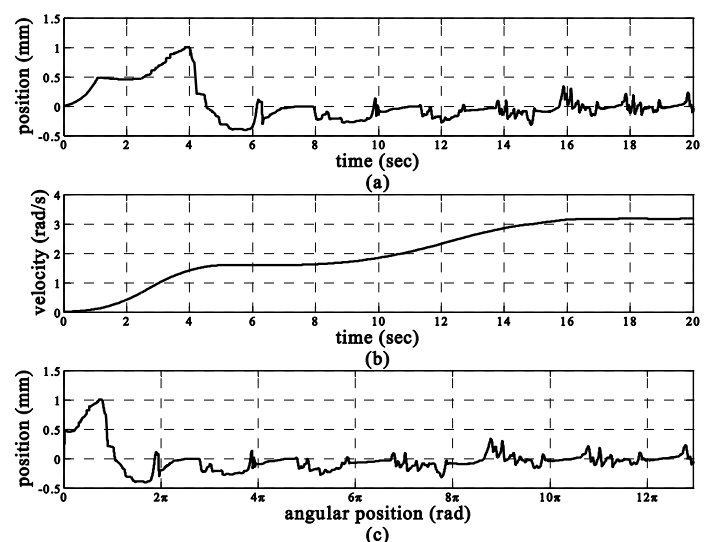

Fig. 11 The experimental results with repetitive controller using position delay and position synchronization. (a) The position response of the load in time domain; (b) the velocity profile of the eccentric wheel; (c) the position response of the load in position domain.

Table 1 The designed control parameters.

\begin{tabular}{|c|c|}
\hline$K_{q}(s)$ & $\frac{157.9127}{s^{2}+17.7714 s+157.9127}$ \\
\hline$T_{q}$ & 0.1125 \\
\hline$T_{b}$ & 0.05 \\
\hline$K_{p}$ & 58 \\
\hline$K_{v i}$ & 9 \\
\hline$K_{v p}$ & 60 \\
\hline$K_{v f}$ & 0.9 \\
\hline$K_{T}$ & 30 \\
\hline
\end{tabular}

